\title{
The Effect of EGFR Inhibitor Treatment in KRAS G13D Mutated Metastatic Colorectal Cancer Background
}

\author{
Thavaneswaran $\mathbf{S}^{1^{*}}$, Shapiro $\mathrm{J}^{2}$, Segelov $\mathrm{E}^{3}$ \\ ${ }^{1}$ Department of Medical Oncology, The Kinghorn Cancer Centre and Garvan Institute of Medical Research, Australia \\ ${ }^{2}$ Department of Medical Oncology, Cabrini Hospital, Melbourne, VIC, Australia \\ ${ }^{3}$ Department of Medical Oncology, Monash Health and Monash University, Australia
}

*Corresponding author: Thavaneswaran S, Department of Medical Oncology, The Kinghorn Cancer Centre and Garvan Institute of Medical Research, Australia Tel: +61 293555655; E-mail: s.thavaneswaran@garvan.org.au

Received date: Mar 23, 2017; Accepted date: Apr 05, 2017; Published date: Apr 12, 2017

Copyright: (c) 2017 Thavaneswaran S. This is an open-access article distributed under the terms of the Creative Commons Attribution License, which permits unrestricted use, distribution, and reproduction in any medium, provided the original author and source are credited.

\begin{abstract}
Cetuximab and panitumumab are monoclonal antibodies directed against the Epidermal Growth Factor Receptor (EGFR) which are now standard treatments for RAS Wild Type (WT) metastatic Colorectal Cancer (CRC), with efficacy in all lines of therapy. The evolution of the use of the EGFR-Inhibitors (EGFR-I) is the landmark journey of the application of a predictive marker and its translation into clinical utility. Here we describe how the evaluation of EGFR-I in patients with the resistant biomarker, i.e., RAS mutant, led to clinical suspicion that the G13D subset of mutated tumours may in fact be an exception. The hypothesis, raised from preclinical data, then retrospective analysis of trial outcomes, was subsequently prospectively tested by our group in a randomised clinical trial (RCT; the ICECREAM study) which unfortunately did not reveal that this particular mutation conferred sensitivity to EGFRI. The investigation of EGFR-I in KRAS G13D mutated metastatic CRC is a good example of the ability of international collaboration to perform RCTs even in rare molecular subtypes, as well as confirming the role of prospective clinical trial evaluation of hypotheses raised by unplanned subgroup analyses.
\end{abstract}

Keywords: Monoclonal antibodies; Epidermal growth factor receptor; Cetuximab; Colorectal cancer

\section{EGFR-inhibitors in Metastatic CRC}

Cetuximab is a chimeric human mouse anti-EGFR monoclonal antibody whilst Panitumumab is a fully humanized monoclonal antibody [1]. Cetuximab and panitumumab recognize similar epitopes targeting the extracellular region of EGFR to effectively inhibit liganddependent downstream signalling [2] but may have a different ability to bind to mutated EGFR as they are different isotopes [3].

Cetuximab first demonstrated efficacy in irinotecan-refractory advanced CRC in the BOND study where patients were retreated with irinotecan plus cetuximab or cetuximab alone [4]. This trial demonstrated single agent activity for cetuximab, which was potentiated (increased response rates and delayed progression) when irinotecan was added to cetuximab, even though all patients had previously progressed on this agent. The overall response rate in the intention-to-treat population was $22.9 \%$ (95\% confidence interval [CI] 17.5-29.1\%) with combination-therapy and $10.8 \%$ (95\% CI, 5.7-18.1\%) with monotherapy $(\mathrm{P}=0.007)$. In those treated with the combination, the median duration of response was 5.7 months compared to 4.2 months with monotherapy. When a more stringent definition was applied for irinotecan resistance-progression during or within one month after irinotecan therapy, the response rates were $25.2 \%$ (95\% CI, $18.1-33.4 \%$ ) and $14.1 \%$ (95\% CI, $7.0-24.4 \%)$ in the combinationtherapy and monotherapy groups, respectively $(\mathrm{P}=0.07)$. However, the number of patients in this comparison were limited $(n=206)$. No Overall Survival (OS) benefit was seen, at least in part attributable to cross over to combination treatment on progression with cetuximab monotherapy. There was no molecular analysis used to select patients nor was there any tissue available to retrospectively interrogate benefit on the basis of tumour RAS status.

In a similar refractory CRC setting, the CO.17 trial randomised patients to cetuximab plus best supportive care versus best supportive care alone. The addition of cetuximab to best supportive care improved OS, Progression Free Survival (PFS) and better maintained quality of life [5]. Again, these results were based on a molecularly unselected population.

\section{KRAS mutational status as a predictive biomarker for EGFR-I benefit}

The practice-changing affirmation that KRAS mutations are a predictive biomarker for cetuximab resistance is based on the retrospective testing of tumours collected in the CO.17 study where a range of biomarkers were explored, including mutations within the $K R A S$ gene [5]. Karapetis et al. [6] demonstrated that the improved outcomes seen with cetuximab therapy were limited to patients with tumours that did not harbour a mutation in exon 2 of the KRAS gene. In patients with $K R A S$ WT tumours, treatment with cetuximab compared with best supportive care alone improved OS (median 9.5 vs. 4.8 months; hazard ratio (HR) for death, 0.55 ; 95\% CI, 0.41-0.74; $\mathrm{P}<0.001)$ and median PFS 3.7 vs 1.9 months; HR for progression or death, $0.40 ; 95 \% \mathrm{CI}, 0.30-0.54 ; \mathrm{P}<0.001$. This is in contrast to no progression-free or overall survival benefit for cetuximab seen amongst those with KRAS mutated tumours; HR for overall survival, 0.98 $(\mathrm{P}=0.89)$ and HR for progression-free survival $0.99(\mathrm{P}=0.96)$. It is important to note that amongst patients who received best supportive care alone, the KRAS mutation status was not prognostic, i.e., with no association with overall survival (HR for death, 1.01; $\mathrm{P}=0.97$ ). 
Immediately preceding [7] this, Amado et al. [8] retrospectively analysed KRAS mutation status from tumour specimens collected in a phase III metastatic CRC trial comparing panitumumab monotherapy to best supportive care. The purpose of this analysis was to evaluate whether the effect of panitumumab on PFS differed by $K R A S$ status. The treatment effect on PFS in patients with KRAS Wild type (WT) tumours was significantly greater, HR 0.45 with $95 \%$ CI, $0.34-0.59$ $(\mathrm{P}<0.0001)$ compared with mutant tumours, HR 0.99, 95\% CI, $0.73-1.36$. This study concluded that the efficacy of panitumumab monotherapy in patients with advanced CRC is limited to those with KRAS WT tumour. Additional retrospective KRAS analyses of panitumumab trials in the first, second and refractory setting were later performed, again demonstrating an improvement in PFS and response rate in patients with KRAS WT metastatic CRC, but not in their mutant KRAS counterparts [7,9,10]. Together with the retrospective $K R A S$ analyses from the cetuximab trials, the American Society of Clinical Oncology published a provisional clinical opinion in 2009 recommending that EGFR monoclonal antibody use be restricted to $K R A S$ exon 2 WT patients. This was rapidly adopted by regulatory agents, and in clinical practice [11].

\section{Combination treatment with irinotecan}

The pivotal BOND study, apart from demonstrating benefit for cetuximab monotherapy, also reported the phenomenon of enhanced outcomes with the addition of irinotecan to cetuximab despite proven progression on irinotecan [4]. The MABEL study, a large multinational evaluation of the efficacy and safety of cetuximab plus irinotecan in patients with EGFR expressing (based on immunohistochemistry, IHC) metastatic CRC in a community practice setting, also confirmed this apparent synergy, again in molecularly unselected patients [12]. Patients were required to have received an irinotecan-containing regimen as their latest line of therapy and have confirmed disease progression on, or within 6 months of this treatment. The primary endpoint of the study was met, PFS at 12 weeks was $61 \%$ compared with the expected PFS rate at 12 weeks, of $50 \%$. The median survival was 9.2 months and the treatment was generally well tolerated. The authors proposed that the addition of cetuximab was able to overcome resistance to irinotecan. It is important to note that these study patients would qualify as unselected, given that EGFR expression based on IHC is no longer deemed a useful predictive biomarker for cetuximab benefit $[4,13-15]$.

\section{Suggestion of EGFR-I benefit with G13D mutations}

The adoption of KRAS exon 2 WT status as an absolute biomarker for response to anti-EGFR monoclonal antibodies was rapidly adopted into clinical practice. However, interest arose as to whether there was an exception to this rule for the specific mutation in KRAS exon 2 of $\mathrm{c}$. 38G.A (p.Gly13Asp), annotated as G13D, a relatively common mutation accounting for approximately $19 \%$ of $K R A S$ mutations [16], with an incidence of 5-8\% of all metastatic colorectal cancers [17].

In vitro studies have demonstrated less activating potential for KRAS G13D mutations compared to codon 12 mutations [18], whilst unplanned subset analyses pooled from large clinical trials appeared to indicate a higher than expected prevalence of G13D mutations in the small group of patients with $K R A S$ mutated tumours who did achieve a sustained response to EGFR-I [19-23].

At least three retrospective studies examining the sensitivity of G13D mutated mCRC were performed. DeRoock et al. [16] retrospectively compared response to EFGR-I of KRAS G13D compared with other KRAS mutations. The dataset was composed of 579 patients pooled from several clinical trials including CO.17, BOND, MABEL, EMR202600, EVEREST, BABEL and SALVAGE. This analysis concluded a longer survival amongst G13D KRAS mutated tumours treated with cetuximab compared to other KRAS mutated tumours. Univariate and multivariate analyses were performed and for patients who received any cetuximab treatment, OS was significantly better for the G13D cohort (and not significantly different to the OS seen in patients with KRAS WT tumours). In the multivariate analysis with G13D taken as the reference, the HR for survival was $0.50(95 \%$ CI, 0.31-0.81; $\mathrm{P}=0.005)$ for other $K R A S$ mutations and 0.94 (0.64-1.48; $\mathrm{P}=0.79$ ) for WT tumours. Interestingly, this survival benefit was not seen amongst the 10 patients who had received cetuximab monotherapy, with all benefit reported in patients receiving cetuximab in combination with irinotecan $(\mathrm{n}=22)$. Similarly, no objective responses were seen in the 10 KRAS G13D mutated tumours treated with cetuximab monotherapy, compared to 2 of 22 patients achieving a partial response with the combination. There was no significant improvement in objective response in G13D mutated tumours compared to other KRAS mutations, although the objective benefit seen with WT tumours only just reached statistical significance, with wide confidence intervals due to low numbers (HR 0.22 (0.05-0.97); $\mathrm{P}=0.04)$.

The prognostic implications of the KRAS G13D mutation was compared to other KRAS mutations based on the best supportive care arm of the CO.17 trial. There was no PFS differences ascertained between G13D, other KRAS mutant or WT tumours. The OS appeared similar between G13D and other KRAS mutations, however there was a clear trend towards a worse prognosis for G13D compared to WT tumours; HR 1.82 (0.99-3.34), $\mathrm{P}=0.053$ with G13D mutations set as the reference $=1.0[16]$.

Tejpar et al. [19] used two randomized trials, OPUS and CRYSTAL, both conducted in the first line metastatic setting using FOLFOX and FOLFIRI respectively, with or without cetuximab to evaluate outcomes on the basis of mutational status. PFS, OS and tumour response all showed significant heterogeneity on the basis of mutational status. While KRAS WT tumours derived clear and significant benefit from the addition of cetuximab to chemotherapy, the other mutational groups had HR and corresponding 95\% CIs all crossing 1, indicating a non-significant difference for the addition of cetuximab to a chemotherapy backbone. In the pooled analysis, KRAS G13D-mutant tumours had a significantly improved response to chemotherapy compared to other mutations (HR 0.40, 95\% CI, 0.18-0.92; $\mathrm{P}=0.032$ ) without a significantly different PFS or OS. The G13D KRAS-mutant tumours treated with cetuximab had an improved PFS, OS and objective response rate compared with G12V mutant tumours. This was not better than the OS or objective responses seen with KRAS WT tumours. This study concluded that "the positive treatment effect observed for patients with KRAS G13D-mutant tumours was a consequence of a combination of the poor prognosis observed for these patients under standard chemotherapy alone, or best supportive care in chemo refractory patients [16] and the improved outcome under treatment with cetuximab" [19].

With regards to panitumumab, similar analyses were performed to test subgroups of RAS mutations. The association between six different KRAS mutations (G12D, G12V, G13D, G12C, G12A, G12S and WT) and outcomes were explored in a pooled analysis of three panitumumab-based studies-20050203 (FOLFOX4+/-panitumumab), 
20050181 (FOLFIRI+/-panitumumab) and 20020408 (best supportive care+/-panitumumab) in first, second and third line therapy, respectively [1]. There were no consistent prognostic differences by mutation type, with the exception of G12C and G12A mutations. Patients with these mutation types treated with best supportive care showed a trend towards worse PFS and a significantly worse OS. KRAS G13D mutations actually appeared to be a negative predictive factor for PFS (HR 1.60; 95\% CI, 1.05-2.46) and OS (HR 2.47; 95\% CI, 1.51-4.03) for the panitumumab-containing arm of the first-line study [9] with only borderline statistical significance on OS after quantitative interaction testing. However, other studies and the pooled analysis did not concur, so that the overall conclusion was that no individual mutant KRAS that was consistently associated with panitumumab treatment effects on PFS or OS, apart from the overall finding of the negative predictive factor for EGFR-I response of KRAS mutations compared to WT.

Mao et al. [24] conducted a systematic review and meta-analysis to address the question of whether patients with KRAS G13D mutations who received cetuximab treatment had better clinical outcomes than metastatic colorectal patients with KRAS codon 12 mutations. Ten studies were identified for inclusion; however a pooled analysis was only possible for objective response rate due to inconsistencies in reporting of PFS and OS endpoints. Among patients who received cetuximab-based treatment, G13D mutations had a higher ORR than KRAS codon 12 mutations (22 vs $16 \%$; RR 1.64; 95\% CI, 1.13-2.38; $\mathrm{P}=0.009)$ although this was significantly less than patients with WT KRAS tumours $23 \%$ vs. $44 \%$ respectively; RR 0.54 (95\% CI, 0.38-0.77; $\mathrm{P}=0.001$ ). The cetuximab benefit for KRAS G13D mutated tumours compared to $K R A S$ codon 12 mutations was not seen in the first-line setting or the cetuximab monotherapy subgroup.

\section{What does the prospective ICECREAM trial add?}

In an editorial in the Journal of Clinical Oncology entitled 'Hurdles and complexities of codon 13 KRAS mutations', Morelli and Kopetz stated that "The gold standard for validation would be a prospective study of EGFR monoclonal antibody in patients whose tumours contain a codon 13 KRAS mutation... however such a study would require a considerable effort to complete"? [25]. The ICECREAM (Irinotecan Cetuximab Evaluation and Cetuximab Response Evaluation among Patients with a G13D Mutation) study is the only clinical trial to prospectively evaluate whether KRAS G13D mutations were indeed unique in their sensitivity to EGFR inhibition and whether there is any further benefit with the addition of irinotecan in the biomarker selected population [26]. In addition to interrogating KRAS G13D as a predictive biomarker for cetuximab benefit, the ICECREAM study also evaluated this treatment in a super-select group of patients with no mutations in KRAS, NRAS, BRAF or PI3KCA 'quadruple wild-type' [27].

The primary endpoint of this phase II trial was progression free survival at 6 months. All patients were required to have chemotherapy - refractory disease - having failed or be intolerant of standard lines of therapy and progression on or within 6 months of irinotecan therapy. Patients were recruited from 14 sites across Australia and one site each in Spain, Italy and England between November 2012 and December 2014. Actual recruitment exceeded the pre-study projection at all times. A total of 53 patients were recruited to the KRAS G13D arm of the study, with treatment assignment stratified by hospital.
To date, only results from the G13D cohort of the ICECREAM study have been published [27]. There was no statistically significant improvement in disease control at 6 months with the use of cetuximab monotherapy or cetuximab plus irinotecan. The 6-month progressionfree survival rate was $10 \%$ (95\% CI, 2-26\%) for cetuximab compared with $23 \%$ (95\% CI, 9-40\%) for the combination with corresponding HR of 0.74 (95\% CI, 0.42-1.32). There were no responses seen among patients who received cetuximab monotherapy $(n=27)$, however there were 2 responses among the 26 patients who received combination treatment. We concluded that the responses observed in those treated with cetuximab plus irinotecan may reflect an effective synergy of the combination although they may also be indicative of persistent irinotecan sensitivity. Although not reaching statistical significance, there were some imbalances in baseline characteristics between treatment arms. Of particular relevance, time since metastatic diagnosis was greater in the cetuximab plus irinotecan arm (28.1 vs 19.1 months) as was the median time since last irinotecan treatment (4.8 vs. 2.8 months). Both of these factors would confer an advantage for those treated with combination treatment-a greater proportion of patients with more indolent disease and an increased possibility of irinotecan sensitivity. The 'quadruple wild-type' arm of the ICECREAM study, due to be published shortly, will assist in differentiating between true synergy of combination treatment and irinotecan re-treatment effects.

\section{Conclusion}

The lack of responses to cetuximab monotherapy in this prospective trial disproved the hypothesis that G13D mutated tumours harbour sensitivity to EGFR-monoclonal antibody therapy and signalled that further investigation was unlikely to yield results which would alter clinical practice. The ICECREAM study highlights the importance of prospective evaluation of hypotheses generated from retrospective analyses. In the era of cancer genomics and personalized medicine, this study provides that it is feasible to conduct clinical trials in rare molecular subtypes through national and international collaboration.

\section{References}

1. Peeters M, Douillard JY, Van Cutsem E, Siena S, Zhang K, et al. (2012) Mutant KRAS Codon 12 and 13 Alleles in Patients With Metastatic Colorectal Cancer: Assessment As Prognostic and Predictive Biomarkers of Response to Panitumumab. J Clin Oncol 31: 759-765.

2. Freeman D, Sun J, Daimases A, Bass R, Jung K, et al. (2008) Panitumumab and cetuximab epitote mapping and in vitro activity. J Clin Oncol 26: 14536

3. Montagut C, Daimases A, Bellosillo B, Crespo M, Pairet S, et al. (2012) Identification of a mutation in the extracellular domain of the epidermal growth factor receptor conferring cetuximab resistance in colorectal cancer. Nat Med 18: 221-223.

4. Cunningham D, Humblet Y, Siena S, Khayat D, Bleiberg H, et al. (2004) Cetuximab monotherapy and cetuximab plus irinotecan in irinotecanrefractory metastatic colorectal cancer. N Engl J Med. 351: 337-345.

5. Jonker D, O'Callaghan C, Karapetis C, Zalcberg J, Tu D, et al. (2007) Cetuximab for the treatment of colorectal cancer. N Engl J Med 357: 2040-2048.

6. Karapetis C, Khambata-Ford S, Jonker D, O'Callaghan C, Tu D, et al. (2008) K-ras Mutations and Benefit from Cetuximab in Advanced Colorectal Cancer. N Engl J Med 359: 1757-1765.

7. Van Cutsem E, Peeters M, Siena S, Humblet Y, Hendlisz A, et al. (2007) Open-label phase III trial of panitumumab plus best supportive care compared with best supportive care alone in patients with chemotherapyrefractory metastatic colorectal cancer. J Clin Oncol 25: 1658-1664. 
8. Amado R, Wolf M, Peeters M, Van Cutsem E, Siena S, et al. (2008) WildType KRAS Is Required for Panitumumab Efficacy in Patients With Metastatic Colorectal Cancer. J Clin Oncol 26: 1626-1634.

9. Douillard JY, Siena S, Cassidy J, Tabernero J, Burkes R, et al. (2010) Randomised, phase III trial of panitumumab with infusional fluorouracil, leucovorin, and oxaliplatin (FOLFOX4) versus FOLFOX4 alone as firstline treatment in patients with previously untreated metastatic colorectal cancer: The PRIME study. J Clin Oncol 28: 4697-4705.

10. Peeters M, Price T, Cervantes A, Sobrero AF, Ducreux M, et al. (2010) Randomized phase III study of panitumumab with fluorouracil, leucovorin, and irinotecan (FOLFIRI) compared with FOLFIRI alone as second-line treatment in patients with metastatic colorectal cancer. J Clin Oncol 28: 4706-4713.

11. Allegra CJ, Jessup JM, Somerfield MR, Hamilton SR, Hammond EH, et al. (2009) American Society of Clinical Oncology provisional clinica opinion: testing for KRAS gene mutations in patients with metastatic colorectal carcinoma to predict response to anti-epidermal growth factor receptor monoclonal antibody therapy. J Clin Oncol 27: 2091-2096.

12. Wilke H, Glynne-Jones R, Thaler J, Adenis A, Preusser P, et al. (2008) Cetuximab Plus Irinotecan in Heavily Pretreated Metastatic Colorectal Cancer Progressing on Irinotecan: MABEL Study. J Clin Oncol 26: 5335-5343.

13. Saltz L, Meropol N, Loehrer PS, Needle M, Kopit J, et al. (2004) Phase II trial of cetuximab in patients with refractory colorectal cancer that expresses the epidermal growth factor receptor. J Clin Oncol 22 1201-1208.

14. Zhang W, Gordon M, Press O, Rhodes K, Vallböhmer D, et al. (2006) Cyclin D1 and epidermal growth factor polymorphisms associated with survival in patients with advanced colorectal cancer treated with cetuximab. Pharmacogenet Genomics 16: 475-483.

15. Wierzbicki R, Jonker D, Moore M, Berry SR, Loehrer PJ, et al. (2008) A phase II multicenter study of cetuximab monotherapy in patients with EGFR-undetectable refractory metasatic colorectal carcinoma. J Clin Oncol 26: 4065

16. De Roock W, Jonker D, Di Nicolantonio F, Sartore-Bianchi A, Tu D, et al. (2010) Association of KRAS p.G13D Mutation with Outcome in Patients with Chemotherapy-refractory metastatic colorectal cancer treated with cetuximab. JAMA 304: 1812-1820.

17. Normanno N, Tejpar S, Morgiollo F, De Luca A, Van Cutsem E, et al. (2009) Implications for KRAS status and EGFR-targeted therapies in metastatic CRC. Nat Rev Clin Oncol 6: 519-527.
18. Guerro S, Casanova I, Farre L, Mazo A, Capella G, et al. (2000) K-ras codon 12 mutation induces higher level of resistance to apoptosis and predisposition to anchorage-independent growtt that codon 13 mutation or proto-oncogene overexpression. Cancer Res. 60: 6750-6756.

19. Tejpar S, Celik I, Schlichting M, Sartorius U, Bokemeyer C, et al. (2012) Association of KRAS G13D Tumor Mutations With Outcome in Patients with Metastatic Colorectal Cancer Treated with First-Line Chemotherapy with or without Cetuximab. J Clin Oncol 30: 1-8.

20. Lievre A, Bachet J, Le Corre D, Boige V, Landi B, et al. (2006) KRAS mutation status is predictive of response to cetuximab therapy in colorectal cancer. Cancer Res: 3992-3995.

21. Moroni M, Veronese S, Bienvenuti S, Marrapese G, Sartore-Bianchi A, et al. (2005) Gene copy number for epidermal growth factor receptor (EGFR) and clinical response to antiEGFR treatment in colorectal cancer. A cohort study. Lancet Oncol 6: 279-286.

22. Bienvenuti S, Santore-Bianchi A, Di Nicolantonio F, Zanon C, Moroni M, et al. (2007) Oncogenic activation of RAS/RAF signaling pathway impairs the response of metastatic colrectal cancers to anti-epidermal growth factor receptor antibody therapies. Cancer Res 67: 2643-2648.

23. Goncalves A, Esteyries S, Taylor-Smedra B, Lagarde A, Ayadi M, et al. (2008) A polymorphism of EGFR extracellular domain is associated with progression free-survival in metastatic colorectal cancer patients receiving cetuximab-based treatment. BMC Cancer 8: 169.

24. Mao C, Huang YF, Yang ZY, Zheng DY, Chen JZ, et al. (2013) KRAS p.G13D Mutation and Codon 12 Mutations Are Not Created Equal in Predicting Clinical Outcomes of Cetuximab in Metastatic Colorectal Cancer. Cancer 119: 714-721.

25. Maria Pia M, Kopetz S (2012) Hurdles and complexities of codon 13 KRAS mutations. J Clin Oncol 30: 3565-3567.

26. Segelov E, Waring P, Desai J, Wilson K, Gebski V, et al. (2016) ICECREAM: randomised phase II study of cetuximab alone or in combination with irinotecan in patients with metastatic colorectal cancer with either KRAS, NRAS, BRAF and PI3KCA wild type, or G13D mutated tumours. BMC Cancer 16: 339.

27. Segelov E, Thavaneswaran S, Waring P, Desai J, Robledo K, et al. (2016) Response to Cetuximab With or Without Irinotecan in Patients with Refractory Metastatic Colorectal Cancer Harboring the KRAS G13D Mutation: Australasian Gastro-Intestinal Trials Group ICECREAM Study. J Clin Oncol 34: 2258-2264. 